\title{
Commentary
}

\section{Who is responsible for the vaccination of migrants in Europe?}

S Hargreaves, LB Nellums, M Ramsay, V Saliba, A Majeed, S Mounier-Jack, JS Friedland

International Health Unit, Section of Infectious Diseases and Immunity, Imperial College London, London, UK (S Hargreaves FRCPE, LB Nellums PhD, Prof JS Friedland FMedSci); Primary Care and Public Health, Imperial College London (Prof A Majeed); Public Health England (M Ramsay, V Saliba); London School of Hygiene and Tropical Medicine, Health Protection Research Unit (S Mounier-Jack MBAAssociate Professor in Health Policy).

A recent report from the World Health Organization exploring provision of immunisation services to migrants and refugees in the WHO European Region ${ }^{1}$ provides a stark reminder that European health services are a long way off adapting to the rapid demographical shift that the region has witnessed in the past two decades, amid unprecedented rises in both internal and external migration. ${ }^{2}$ Migrants are more likely to be under-immunised - putting them at increased risk of vaccine-preventable diseases currently circulating in Europe - and face greater disease, disability, and deaths from vaccine-preventable diseases. ${ }^{1,3-7}$ Despite this, the WHO report found that less than a third of countries in the region had specific directives on immunisation of migrants in their national programmes, with striking variations in terms of policy, guidance, and implementation of vaccination in migrants, a finding supported by other studies. ${ }^{8,9}$ Data published this month by the ECDC ${ }^{10}$ has shown that measles cases in Europe trebled in 2017, with large outbreaks currently ongoing in several countries, mainly due to suboptimal vaccination coverage, but also linked to cross-border migration within the region of migrants moving to and from large epidemics. Yet there remains a lack of clarity on what vaccinations should be given or re-administered to migrants, and what cost-effective approaches will improve coverage.

Ensuring high levels of vaccination coverage is a key priority of the European Vaccine Action Plan, ${ }^{11}$ whereby all WHO Europe Member States have committed to eliminating endemic 
measles and rubella (>95\% coverage with the measles mumps rubella [MMR] vaccine), sustaining polio-free status, and controlling hepatitis B infection. Yet consistently high levels of migration across the region, coupled with low national uptake in many countries, poses a challenge to achieving this. Importantly, adolescent (age 10-18 years) and adult migrants, in particular, are often excluded from initiatives to assess immunisation status and to offer appropriate catch-up vaccination on arrival, with national policies largely focussed on children under the age of $5 .{ }^{8} 45 \%$ of measles cases reported in Europe in $2017^{10}$ (migration status not reported) were aged 15 years and older, highlighting the need to identify and catch-up those who missed out on routine vaccination in childhood. In a recent Danish study of 2126 asylum seekers $48 \%$ of adolescents (aged 12-18 years) were recorded as unimmunised or status unknown ${ }^{12}$. Undocumented migrants may also be excluded from catch-up vaccination because of significant barriers to healthcare and a lack of entitlement to free statutory health services $^{1,13}$, with $10(31 \%)$ of EU/EEA countries we surveyed reporting that they were charging undocumented migrants for vaccinations. ${ }^{8}$

Existing data show that newly arrived migrants to Europe have lower rates of vaccine coverage than the host populations on arrival, and may have an uncertain vaccination status, including incomplete vaccination history and/or missing documentation of previous vaccinations ${ }^{1,4,5,7,12-14}$; concerns echoed in minority mobile groups such as the Roma community. Outbreaks of infectious diseases such as measles and hepatitis A have been documented specifically in non-EU migrant populations in Europe ${ }^{5,15-17}$. Studies from Sweden and the UK have highlighted that migrant women are less likely than native women to be immunised for rubella. ${ }^{18,19} \mathrm{~A}$ third of asylum-seeking children and adolescents in one study were not immunised in accordance with national guidelines, with Afghans and Eritreans least likely to be up to date, highlighting a need to promote and target vaccination initiatives in this group. ${ }^{12}$ Compounding these issues are migrants' exposure to key social determinants including poor living conditions, low socioeconomic status, and disparities in access to health services on arrival due to language barriers, inability to pay, cultural beliefs, and fear of discrimination. ${ }^{1,13}$ There are additional challenges to administering the full schedule of vaccinations, which is complex and requires multiple appointments, in what are often mobile and diverse populations. Yet if we can get this right, improving coverage of low-cost vaccinations is considerably more favourable than the high costs involved in mounting an 
outbreak response once transmission is established, and treating individuals with vaccinepreventable diseases and their complications.

Latest European guidelines state that migrants should be vaccinated without unnecessary delay according to the schedule of the country in which they intend to stay for more than a week, with priority given to MMR and polio vaccines, and that refugees and asylum seekers should have non-discriminatory, equitable access to vaccination irrespective of their legal status. ${ }^{20}$ However, it is clear that these guidelines are yet to be adopted by many countries and that implementation in practice is challenging, and multiple questions remain around how best to organise and deliver services. For example, how can we best avoid duplicating efforts for the thousands of migrants who transit across numerous countries before they settle, avoiding re-vaccination through better documentation and cross-border initiatives? Should migrants be offered specific vaccines, such as the hepatitis B vaccination by virtue of their increased risk, and should any of the vaccines be mandatory?

As is the case in many areas of migrant health, the evidence-base to help policy makers understand how best to improve outcomes in migrants are notably absent, and more research is needed on cost-effective and acceptable interventions. In addition, the consequences of doing nothing need to be clearly articulated. Underpinning this is a need to address shortfalls in routine data collection across Europe and so steps need to be taken to better estimate immunisation coverage in migrant populations as well as capturing the burden of vaccine-preventable diseases and outbreaks in this group. WHO, UNHCR, and UNICEF recommend that migrants should have full access to prevention services (including vaccination) and high quality care irrespective of their legal status and that tailored immunisation services should be made available for migrants with suboptimal coverage ${ }^{20}$, supported by strong communication and social mobilisation. In addition to improve migrants' knowledge of the receiving health care system, targeted interventions such as door-to-door vaccination initiatives, media campaigns, peer-to-peer interactions - importantly, involving migrants in the planning - may improve vaccine uptake. ${ }^{1}$ Similarly, greater emphasis needs to be placed on raising awareness and knowledge around the unique needs of migrants among front-line health-care professionals tasked with meeting the health needs of this 
group. Migrants are often proactive about their health, and immunisation uptake may well be high if offered in a culturally competent environment.

Vaccine-preventable diseases carry a high morbidity and mortality and the efficacy of vaccine programmes rely on protection of both the individual and maintaining high levels of herd immunity. With global migration at unprecedented levels, failure to address vaccination shortfalls in migrant populations risks undoing years of gain from well-established national vaccine programmes. A renewed focus is now needed to ensure we realise target 3.8 of the Sustainable Development Goal on health to provide "access to safe, effective, quality, and affordable essential medicines and vaccines for all" in European and other destination countries. Policymakers, researchers, and health-care professionals have a responsibility to ensure health systems adapt and develop to an increasingly mobile world and facilitate equitable access to vaccines for all.

\section{References}

1. De Vito E PP, de Waure C, Proscia A, Ricciardi W. A review of evidence on equitable delivery, access and utilization of immunization services for migrants and refugees in the WHO European Region. Copenhagen: WHO Regional Office for Europe, 2017.

2. IOM. World Migration Report 2018. Geneva: IOM, 2018.

3. Pavli DA MD. Health problems of newly arrived migrants and refugees in Europe. $J$ Trav Med 2017; 24(4).

4. Pavlopoulou ID ea. Clinical and laboratory evaluation of new immigrant and refugee children arriving in Greece. BMC Pediatr 2017; 17(1): 132.

5. Mipatrini D SP, Severoni S, et al. Vaccinations in migrants and refugees: a challenge for European health systems. A systematic review of current scientific evidence. Pathog Glob Health 2017; 111(2): 1-2.

6. Vaidya V HN, Joshi A, et al. Immunization status of underfive children in migrants from periurban areas of Pune. Natl J Community Med 2013; 4(3): 457-60.

7. Prymula R SJ, Chlibek R, et al Vaccination in newly arrived immigrants to the European Union. Vaccine 2017; 8 June 2017.

8. Hargreaves S NL, Ravensbergen SJ, et al. Divergent approaches in the vaccination of recently arrived migrants to Europe: a survey of national experts from 32 EU/EEA countries. Euro Surveill (In press).

9. Riccardo F DM, Kojouharova M, Fabiani M, Alfonsi V, et al. Migrant's access to immunization in Mediterranean countries. Health Policy 2012; 105(1): 17-24.

10. ECDC. Measles cases in the EU treble in 2017, outbreaks still ongoing. Stockholm: ECDC; 9th February 2018. 
11. WHO. European Vaccine Action Plan 2015-2020. Geneva: WHO Regional Office for Europe, 2014.

12. Nakken CS SM, Nellums LB, Friedland JS, Hargreaves S, Norredam M. Vaccination status and needs of asylum-seeking children in Denmark: a retrospective data analysis. Public Health 2018; Public Health, in press.

13. Aldridge RW MA, Jakubowski B, et al. Falling through the Cracks: The failure of Universal Healthcare Coverage in Europe London: European Network to Reduce Vulnerabilities in Health, 2017.

14. de la Fuente IG WN, Siegrist C-A, et al. Tetanus immunity as a surrogate for past diphtheria-tetanus-pertussis immunization in migrant children. Paediatr Infect Dis 2013; 32: 274-7.

15. Williams GA BS, Shadwick R, et al. Measles among migrants in the European Economic Area. Scand J Pub Health 2016; 44: 6-13.

16. Jones G HS, Merlin B, et al. Measles outbreak in a refugee settlement in Calais, France: january to February 2016. EuroSurveill Bull Eur sur les Mal Transm 2016; 21(11): 30167.

17. Mellou $\mathrm{K}$ ea. Hepatitis $\mathrm{A}$ among refugees, asylum seekers and migrants living in hosting facilities, Greece, April to December 2016. Euro Surveill 2016; 22(4): 30448.

18. Kakoulidou M ea. Serum levels of rubella-specific antibodies in Swedish women following three decades of vaccination programmes. Vaccine 2010; 28: 1002-7.

19. Hardelid P C-BM, Williams D, et al. Rubella seroprevalence in pregnant women in north Thames: estimates based on newborn screening samples. J Med Screen 2009; 16: 1-6. 20. WHO-UNHCR-UNICEF. Joint technical guidance: general principles of vaccination of refugees, asylum-seekers and migrants in the WHO European Region, 2016. 